\title{
Is There a Need for Contrast Administration Prior to CT-Guided Cervical Nerve Root Block?
}

W e read with great interest the recent article by Miller et $\mathrm{al}^{1}$ regarding lateral decubitus positioning for cervical nerve root block by using CT image guidance to minimize effective radiation dose and procedural time.

The technique of cervical foraminal injection is outlined in the "Materials and Methods" section. The authors state, "A slow $1-\mathrm{mL}$ injection of iohexol diluted in 1-mL 1\% lidocaine was used in all cases to identify inadvertent direct vessel puncture." We ask the authors in how many cases did they identify inadvertent direct vessel puncture with the aid of contrast?

From the $>1000$ procedures we have performed by using the technique outlined in this article, we have never identified vessel puncture with contrast CT. The reason for this is likely to be 2-fold: First, should the contrast be injected intravascularly, it is likely to be washed away by the time CT is performed. Second, it is possible that the given vessel enters the cord at a different level and is therefore not imaged.

In this sense, we believe that contrast administration gives the radiologist a false sense of security. Real-time imaging such as digital subtraction angiography would be needed to reliably exclude inadvertent direct vessel puncture. However, we believe that such measures are also unnecessary on the basis of current best evidence in the literature (case series, ${ }^{2}$ animal experimentation, ${ }^{3}$ and in vitro microscopy ${ }^{4}$ ). Dexamethasone sodium phosphate is likely safe if inadvertently injected intravascularly.

On this basis, we propose that this procedure can be made even safer in 2 ways: By eliminating the administration of contrast, the possibility of an adverse reaction is avoided. Furthermore, the number of imaging series could be reduced to give an even lower effective radiation dose and a shorter procedural time.

\section{REFERENCES}

1. Miller TS, Fruauff K, Farinhas J, et al. Lateral decubitus positioning for cervical nerve root block using CT image guidance minimizes effective radiation dose and procedural time. AJNR Am J Neuroradiol 2013;34:23-28

2. Scanlon GC, Moeller-Bertram T, Romanowsky SM, et al. Cervical transforaminal epidural steroid injections: more dangerous than we think? Spine 2007;32:1249-56

3. Okubadejo GO, Talcott MR, Schmidt RE, et al. Perils of intravascular methylprednisolone injection into the vertebral artery: an animal study. J Bone Joint Surg Am 2008;90:1932-38

4. Derby R, Lee SH, Date ES, et al. Size and aggregation of corticosteroids used for epidural injections. Pain Med 2008;9:227-34

T.M. Ryan

E.C. Kavanagh

P.J. MacMahon

Department of Radiology Mater Misericordiae University Hospita

Dublin, Ireland 